\title{
Antigenic Heterogeneity Associated with Pilus Aggregation and Autoagglutinability in Neisseria gonorrhoeae
}

\author{
By C. W. PENN,* N. J. PARSONS, D. R. VEALE AND H. SMITH \\ Department of Microbiology, University of Birmingham, \\ P.O. Box 363, Birmingham B15 2 TT
}

(Received 27 March 1980; revised 16 June 1980)

\begin{abstract}
A type-specific antigen of Neisseria gonorrhoeae was previously demonstrated by twodimensional immunoelectrophoresis, and was produced by strains adapted to growth in subcutaneous chambers in guinea pigs. This antigen was also present in 'smooth' (nonautoagglutinating) variants selected directly from the first agar cultures of three clinical isolates, without chamber passage. The type-specificity of this antigen paralleled the reactions of pili in immune electron microscopy, suggesting that the type-specific antigens were pili. However, 'rough' (autoagglutinating) variants lacking this type-specific antigen were nevertheless pilated. Examination of one strain by immune electron microscopy showed that the pili of the rough variant differed antigenically from those of the smooth variant. Pili on the rough variant tended to form extensive parallel aggregates, whereas pili on the smooth variant radiated individually from the gonococci. This physical difference might relate to the behaviour of the gonococci in suspension. The significance of pilus variation in immunity to gonococcal infection is discussed.
\end{abstract}

\section{INTRODUCTION}

Gonococcal pili have been associated with colony types T1 and T2 (Jephcott $e$ t al., 1971; Swanson et al., 1971) which are putatively virulent for man (Kellogg et al., 1963). Pili from different strains have been shown, by immune electron microscopy (Novotny \& Turner, 1975) and by antibody-binding studies (Buchanan, 1978), to be antigenically heterogeneous. Although Brinton et al. (1978) demonstrated minor common antigenic determinants and proposed the use of gonococcal pilus antigens as vaccines, Turner \& Novotny (1976), Buchanan et al. (1977) and Robertson (1979) examined the role of pilus antibody in protection against laboratory animal infection and failed to find any striking protective activity. A type-specific antigen (antigen $a$ ) (Penn et al., 1978; Parsons et al., 1979), associated with gonococci adapted to growth in vivo in the guinea pig subcutaneous chamber model, contributed, with other antigen(s), to immunity against chamber infection with the smooth strain BS4 (Parsons et al., 1979). Antigen $a$ now appears to be pili, despite earlier conclusions to the contrary which were based on the assumption that one strain produced only one type of pilus (Penn et al., 1978).

In published electron micrographs, negatively stained pili in gonococcal cultures are distributed in two ways: either well separated and radiating from gonococci (Swanson et al., 1971; Novotny \& Turner, 1975; Arko et al., 1976; McGee et al., 1976) or in parallel aggregates (Jephcott \& Reyn, 1971; Novotny \& Turner, 1975; Novotny et al., 1975). The formation of pilus aggregates may be affected by the ionic strength (Robertson et al., 1977) or the pH (Swanson et al., 1971; Buchanan et al., 1973) of the suspending media, or by strain variability. We now describe differences in pilus aggregation under identical physical conditions in two variants selected in vivo from the same parent strain, and show that these 
differences in aggregation are related to antigenic differences and autoagglutinability in liquid medium.

During preparation of this work (originally described by Penn et al., 1979, in a preliminary communication) for publication, two further reports of gonococcal pilus heterogeneity among variants of individual strains have appeared (Lambden et al., 1980; Salit et al., 1980).

\section{METHODS}

Organisms. Neisseria gonorrhoeae strains BS3, BS4, CS3 and ES3 were passaged three or four times in guinea pig chambers (Penn et al., 1976, 1978), stored in liquid nitrogen, and the first subculture on agar, BS4 (agar) etc., was used in this work. Other variants derived from strain BS (Veale et al., 1975) were obtained as follows. BS4R was a rough variant lacking antigen $a$, derived from BS4 by isolation from a chamber on a guinea pig immunized with the $a$ antigen-antibody complex and challenged with BS4 (Parsons $e$ t al., 1979). The initial culture of BS4R contained a small proportion of smooth organisms, capable of stimulating an immune response to $a$ antigen in rabbits. Pure rough (BS4PR) cultures were obtained by prolonged vortexing of dilute suspensions of BS4R before plating and subculture of resulting single colonies (Parsons et al., 1980). Pure cultures of the smooth variant BS4 (agar) were obtained by the same method. Strain BSDH was selected in vitro from laboratory strain BS, as described by Penn et al. (1977). Strains $\alpha, \beta, \gamma$, $\delta$ and $\epsilon$ were obtained from the urethrae of male patients with acute purulent gonorrhoea, with the assistance of Dr J. C. Clay and her staff at Birmingham General Hospital. Samples of urethral exudate were suspended in $1 \mathrm{ml} 1 \%(\mathrm{w} / \mathrm{v}$ ) Proteose Peptone no. 3 (Difco) containing $8 \%$ (v/v) glycerol (Ward \& Watt, 1971), and several portions were frozen in liquid nitrogen.

Media. The organisms were grown on transparent AG agar (Veale et al., 1975), containing $3.6 \%(\mathrm{w} / \mathrm{v})$ G.C. agar base (Oxoid), $1 \%(\mathrm{w} / \mathrm{v})$ yeast extract (Oxoid), $0 \cdot 7 \%(\mathrm{w} / \mathrm{v})$ glucose and $10 \%(\mathrm{v} / \mathrm{v})$ newborn calf serum (Flow), and incubated in candle extinction jars for $24 \mathrm{~h}$ at $37^{\circ} \mathrm{C}$.

Autoagglutinability in suspension. Agar cultures (one loopful) were suspended in $0.2 \mathrm{ml}$ Trypticase Soy Broth (TSB; BBL) or $\mathrm{NaCl}(0.15 \mathrm{M})$ in tubes $(1 \times 10 \mathrm{~cm})$ to give approximately $10^{10}$ organisms $\mathrm{ml}^{-1}$, and rapidly vortex mixed for 15 to $30 \mathrm{~s}$. Tubes were then allowed to stand for 1 to $2 \mathrm{~min}$, tilted and examined from below, with transillumination by a $60 \mathrm{~W}$ desk lamp, for macroscopic gonococcal aggregation. In suspensions of rough cultures, this was easily seen and often the suspending medium became almost clear as the aggregates settled. In suspensions of smooth cultures, uniform dense turbidity without macroscopic aggregates was maintained for at least 5 min.

Antisera. Rabbit antisera to strains BS3 (agar), CS3 (agar) and ES3 (agar) were prepared as described previously (Penn et al., 1978). Specific antisera to the $a$ antigen-antibody complex were produced in guinea pigs and rabbits as described by Parsons et al. (1979). Antisera to strains $\alpha, \beta, \gamma, \delta$ and $\epsilon$ were obtained from rabbits immunized with primary cultures scraped from the agar surface with a wire loop, avoiding contaminant colonies which comprised 1 to $10 \%$ of the total. Suspensions of gonococci were prepared and diluted in TSB to give $10^{8}$ organisms $\mathrm{ml}^{-1}$ (visual count using a counting chamber) and immediately injected intravenously into rabbits. Successive doses of $0 \cdot 1,0 \cdot 2,0.4$ and $1.6 \mathrm{ml}$ were given at 4 to $5 \mathrm{~d}$ intervals and animals were bled $10 \mathrm{~d}$ after the last injection.

Two-dimensional immunoelectrophoresis (2DIEP). This technique was as described by Penn et al. (1978), using surface wash preparations (Penn et al., 1976) and the antisera described above.

Electron microscopy. The presence and morphology of pili was assessed by examination of negatively stained organisms. Organisms from agar culture sufficient to produce visible turbidity were suspended with a wire loop in distilled water. The suspension was mixed with an equal volume of $3 \%(\mathrm{w} / \mathrm{v})$ potassium phosphotungstate $\mathrm{pH} 7.2$ and applied to Formvar-coated copper grids. Excess fluid was removed by capillary attraction on to filter paper. The grids were dried in air and examined in a Philips EM201 electron microscope at $80 \mathrm{kV}$. Immune electron microscopy (IEM) was based on the method of Almeida \& Waterson (1969): $0 \cdot 1 \mathrm{ml}$ of a suspension of fresh agar-grown gonococci in TSB $\left(10^{10}\right.$ organisms ml-1) was added to $0.8 \mathrm{ml}$ phosphate buffered saline pH 7.2 (PBS; $73 \mathrm{~mm}-\mathrm{NaCl}, 18 \mathrm{~mm}-\mathrm{KH}_{2} \mathrm{PO}_{4}, 57 \mathrm{~mm}-\mathrm{Na}_{2} \mathrm{HPO}_{4}$ ) and $0.1 \mathrm{ml}$ rabbit antiserum. The mixture was incubated at $37^{\circ} \mathrm{C}$ for $30 \mathrm{~min}$, at $0{ }^{\circ} \mathrm{C}$ for $1 \mathrm{~h}$ and centrifuged at $12000 \mathrm{~g}$ for $20 \mathrm{~min}$ at $4{ }^{\circ} \mathrm{C}$. The deposit was washed in $10 \mathrm{ml}$ PBS, the centrifugation was repeated, and PBS was drained off by inversion of the tube for several minutes. The pellet was resuspended in distilled water and negatively stained as described above. 


\section{Table 1. Cross-reactions of a antigens in 2DIEP and pili in IEM}

For each strain, the left-hand column shows the $a$ antigen reaction revealed by 2DIEP*, and the right-hand column shows the pilus reaction in IEM $\dagger$.

\begin{tabular}{|c|c|c|c|}
\hline \multirow[b]{2}{*}{$\begin{array}{c}\text { Antiserum } \\
\text { to } \\
\text { strain: }\end{array}$} & \multicolumn{3}{|c|}{ Chamber-adapted strains } \\
\hline & $\begin{array}{c}\text { BS3 } \\
\text { (agar) }\end{array}$ & $\begin{array}{c}\text { CS3 } \\
\text { (agar) }\end{array}$ & $\begin{array}{c}\text { ES3 } \\
\text { (agar) }\end{array}$ \\
\hline BS3 (agar) & ++ & -- & - \pm \\
\hline CS3 (agar) & -- & ++ & $-\quad-$ \\
\hline ES3 (agar) & -- & - \pm & ++ \\
\hline$\gamma$ & - ND & $-\mathrm{ND}$ & - ND \\
\hline$\delta$ & $-\mathrm{ND}$ & -- & -- \\
\hline $\begin{array}{l}\epsilon \\
\text { BS4 (agar) } \\
\text { Antigen } a \\
\text { BS4PR }\end{array}$ & - ND & $-\mathrm{ND}$ & $-\mathrm{ND}$ \\
\hline
\end{tabular}

\begin{tabular}{|c|c|c|c|c|}
\hline Sm & $\begin{array}{l}\mathrm{h} \text { clon } \\
\text { tan iso }\end{array}$ & & $\begin{array}{l}\text { Var } \\
\text { str }\end{array}$ & $\begin{array}{l}\text { ts of } \\
\text { BS }\end{array}$ \\
\hline$\gamma$ & $\delta$ & $\epsilon$ & $\begin{array}{c}\text { BS4 } \\
\text { (agar) }\end{array}$ & BS4R \\
\hline-- & -- & -- & & \\
\hline-- & -- & -- & & \\
\hline-- & -- & -- & & \\
\hline++ & ++ & - \pm & & \\
\hline+ \pm & ++ & - \pm & & \\
\hline- \pm & - \pm & $+\overline{+}$ & & \\
\hline & & & ++ & \\
\hline & & & ++ & - \pm \\
\hline & & & - \pm & - \pm \\
\hline
\end{tabular}

$*+$ Presence of $a$ antigen precipitate; - , absence of $a$ antigen precipitate.

$\uparrow+$, Pili heavily coated with antibodies; \pm , pili lightly coated with antibodies; - , pili free of antibodies; ND, not determined.

\section{RESULTS}

\section{Cultural character and $a$ antigen formation in clinical isolates}

The five isolates $\alpha, \beta, \gamma, \delta$ and $\epsilon$ all formed predominantly small colonies on primary culture, produced pili, and formed rough suspensions. When surface washes were examined by 2DIEP with homologous rabbit antisera, lines of precipitate corresponding in shape, density and position to the $a$ antigen precipitate were not seen, except possibly a weak reaction of the $\delta$ strain. Isolated colonies of each strain were subcultured separately, and the resultant clones were examined for rough or smooth character. Smooth clones were not detected among 30 clones tested of strains $\alpha$ and $\beta$, but they were found in strains $\gamma(3 / 10)$, $\delta(3 / 12)$ and $\epsilon(2 / 16)$. These smooth clones were pilated, and produced $a$ antigen precipitates in 2DIEP (Parsons et al., 1979).

\section{Cross-reactions by 2 DIEP and IEM of three guinea pig-adapted strains and smooth clones selected from three clinical isolates}

In IEM tests, reactions of varying intensities were observed using different sera. Reactions were classed as positive if the pilus structure was completely masked by a 'fuzzy coat' of adherent antibody molecules (e.g. Fig. 1a). Intermediate $( \pm$ ) reactions were those in which the pilus structure was distinguishable, with antibody molecules attached at intervals, often giving a ladder-like structure with pili held at a constant distance apart by cross-linking antibody (e.g. Fig. 1c), as described by Novotny et al. (1975). Reactions were negative if no or negligible antibody was seen attached to the pili, and pili in such preparations appeared similar to those in Fig. 2(a). Results of cross-reactions between strains are summarized in Table 1. Whenever the IEM reaction was positive, an $a$ antigen precipitate was seen in 2DIEP, indicated by + in the left-hand columns in Table 1. When IEM was negative, $a$ precipitates were absent (indicated by - in the left-hand columns in Table 1), but in some cases intermediate IEM reactions were seen, which did not correspond with $a$ precipitates. Thus, the presence of $a$ precipitates correlated with strongly positive IEM reactions of pili. Five $a$ antigen serotypes were present in this group of six strains: BS4 (agar), CS3 (agar), ES3 (agar), $\gamma / \delta$, and $\epsilon$. 

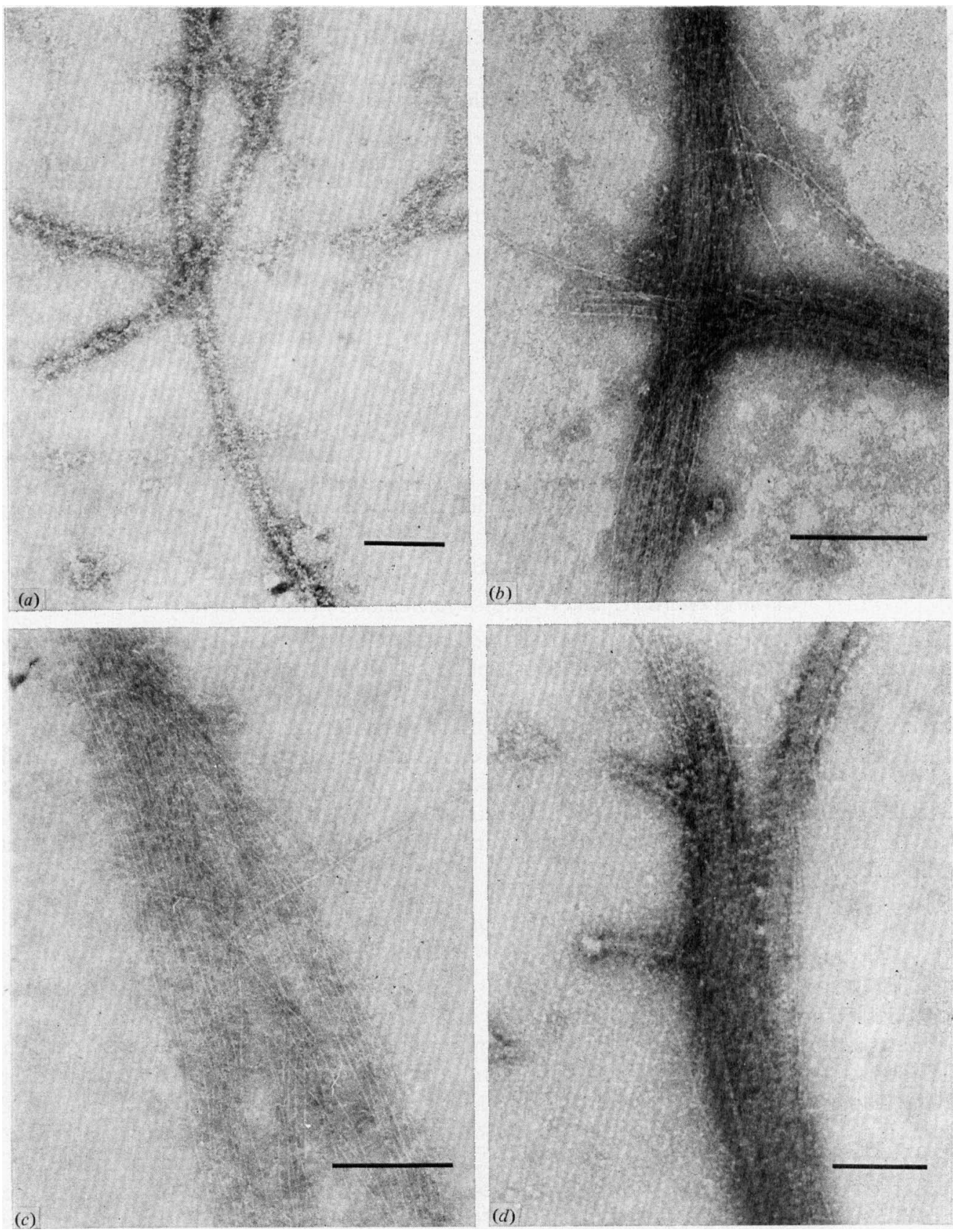

Fig. 1. IEM reactions of pili of rough and smooth variants: (a) BS4 (agar) pili with homologous antiserum, an example of a positive ( + ) reaction; $(b)$ BS4R pili with antiserum to strain BS4 (agar); (c) BS4 (agar) pili with antiserum to strain BS4PR, an example of an intermediate ( \pm ) reaction; (d) BS4R pili with antiserum to strain BS4PR. Negative stain. Bar markers represent $0.2 \mu \mathrm{m}$.

\section{Comparison by 2 DIEP and IEM of rough and smooth variants of strain BS}

The $a$ precipitate in 2DIEP was originally seen when surface washes of strain BS4 (agar) reacted with rabbit antiserum to live homologous organisms (Penn et al., 1978; Parsons et al., 1979). In IEM, the pili of the smooth variant BS4 (agar) reacted strongly with this antiserum (Fig. $1 a$ ), and with antiserum specific for the $a$ antigen prepared by injecting 

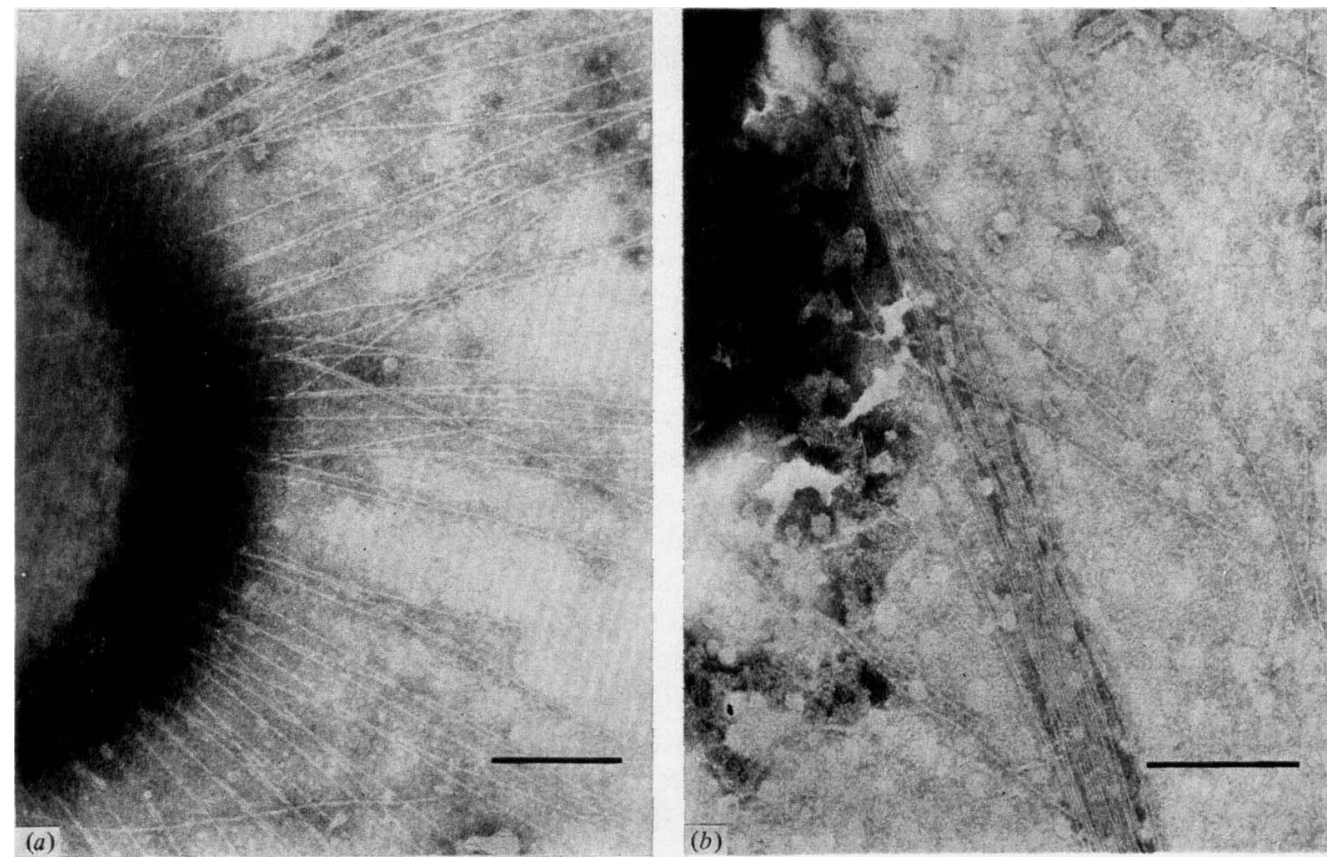

Fig. 2. Electron micrographs of pili in agar-grown cultures suspended in distilled water before negative staining: (a) strain BS4 (agar) pili radiating separately from a cell; (b) strain BS4R pili, many showing lateral aggregation. Bar markers represent $0 \cdot 2 \mu \mathrm{m}$.

guinea pigs or rabbits with excised $a$ immunoprecipitates. Surface washes of the rough variant BS4R formed no $a$ precipitates in 2DIEP with antisera either to whole smooth organisms or to $a$ immunoprecipitates, but pili from this variant gave intermediate reactions with these antisera (Fig. 1b). Like BS4R, strain BSDH surface washes did not form $a$ precipitates, and IEM reactions of pili were similar to those of BS4R. Antiserum from rabbits immunized with BS4PR did not form $a$ precipitates with BS4 (agar) surface washes, but gave intermediate IEM reactions both with pili from BS4 (agar) (Fig. 1c) and with homologous pili (Fig. 1d). The latter reaction appeared stronger than the former, but was difficult to assess due to spontaneous aggregation of pili. These results are summarized in Table 1 .

\section{Physical character of pili from rough and smooth variants}

Cultures of strain BS4 (agar) suspended in distilled water and immediately negatively stained (no centrifugation) always showed pili with minimal aggregation radiating from organisms (Fig. 2a). In contrast, cultures of strain BS4R, prepared similarly, contained many extensive lateral aggregates of pili (Fig. $2 b$ ), sometimes detached from organisms; this effect was accentuated by centrifugation during preparation of cultures for IEM, and was observed in control preparations of BS4R treated with normal rabbit serum. Pili of strain BSDH also formed aggregates, but they were seen less frequently than in strain BS4R.

\section{DISCUSSION}

The correlation between detection of antigen $a$ in 2DIEP and strong reactions of pili in IEM (Table 1) strongly indicated that the antigen was pilus-associated. Previously, we inferred that this antigen was not pili, since it was not detected in the pilated strain BSDH (Penn et al., 1978). However, the evidence for antigenic variation of pili among variants of the BS strain invalidates the inference. 
We have used the terms 'smooth' and 'rough' to describe non-autoagglutinating and autoagglutinating cultures, respectively, according to the nomenclature of Wilson \& Miles (1975). Previously, autoagglutination of gonococcal cultures has been associated with the pilated colony types $\mathrm{T} 1$ and $\mathrm{T} 2$, whereas non-pilated colony types T3 and T4 did not strongly autoagglutinate (Kellogg et al., 1963; Jephcott \& Reyn, 1971; Jephcott et al., 1971; Swanson et al., 1971). In contrast, we describe here the smooth character of some pilated strains. Autoagglutination is often associated with pilation in many bacterial species (Brinton et al., 1978), but a correlation between the presence of a particular pilus type and the absence of autoagglutination has not been reported. The exact role of pili in these phenomena is not clear. Mutual repulsion of pili from adjacent cells of smooth cultures might prevent autoagglutination, but there was little evidence from electron microscopy that aggregation of pili from adjacent cells mediated autoagglutination of rough cultures, although preparation of cultures for electron microscopy might have disrupted any such linkages. An alternative explanation for rough-smooth variation is that differences in outer membrane proteins between variants are responsible for differences in autoagglutinability. However, the very strong correlation between expression of pilus antigen $a$ and smooth behaviour in suspensions of the six strains described argues against this, unless there is a close genetic linkage between genes for pilus antigens and for outer membrane proteins, leading to co-variation. Preliminary observations of the outer membrane protein content of surface washes by polyacrylamide gel electrophoresis (Laemmli, 1970) failed to show differences between variants BS4 (agar) and BS4R, although this possibility cannot yet be excluded.

The correlation between smooth character and possession of antigen $a$ was first noticed during subculture of single colonies of a strain (BS4) adapted to growth in guinea pig chambers (Parsons et al., 1979). Provided small, pilated colony types were selected for subculture, smooth character of the resulting clones was a reliable marker for the presence of pilus antigen $a$. (Large, non-pilated colony types T3 or T4 would also give smooth clones, but these would not possess antigen $a$.) By this means antigen $a$-positive clones were identified in clinical isolates, and comprised a minority (10 to $30 \%$ ) of clones in three of five isolates examined; thus their significance in human infection may not be great. However, the $a$ determinant of pili appears to be immunologically potent, since, when antisera to these clinical isolates were raised against the primary cultures unselected for antigen $a$, ample antibody responses to the $a$ antigens occurred. This phenomenon was also apparent when antisera were raised against strains BSDH or BS4R; in neither strain could antigen $a$ be detected, yet antibody to it was induced, presumably by a small minority of antigen $a$-positive organisms (Parsons et al., 1980).

Antigen $a$ appears to provide another example of antigenic diversity of pili between strains which is well documented (Novotny \& Turner, 1975; Brinton et al., 1978; Buchanan, 1978). The existence of minor, common antigenic determinants on pili of different serotypes has also been reported (Brinton et al., 1978) and it is probably responsible for the intermediate reactions between pili in IEM, which do not correlate with formation of an $a$ immunoprecipitate. Antigenic variants of pili within a strain have not been described in detail, although Novotny \& Turner (1975) noted antigenic heterogeneity of pili within a culture, revealed by IEM. The variants described here might be separate strains derived from a mixed culture at initial isolation. This is unlikely in the case of the variants of the laboratory strain BS, which have been investigated here in detail. Strain BS had been selectively subcultured for small colony type from single colonies many times in vitro, before serial passage in guinea pig chambers. However, clinical isolates $\gamma, \delta$ and $\epsilon$ were not subcultured extensively, and their 'smooth variants' might have represented separate strains present as a minority in the original isolates.

Physical heterogeneity of purified pili from the same strain, which separated into two bands on isoelectric focusing, was reported by Robertson et al. (1977), and colony opacity 
variants which produced pili differing physically have also been described (Salit \& Gotschlich, 1978; Salit et al., 1980; Lambden et al., 1980). Aggregation differences in pili between variants of strain BS suggested similar physical heterogeneity of pili, but there were no detectable differences in colony morphology or opacity between variants BS4 (agar) and BS4R, both of which formed small, 'double highlight' (DH) colonies (Penn et al., 1977).

The existence of antigenic variant pilus types within a strain has important implications for gonococcal immunity. Gonococcal pili have been proposed as the basis for a vaccine for human use (Brinton et al., 1978), and we have shown that antigen $a$ of strain BS4 (agar) contributes to immunity (Parsons et al., 1979). However, on challenge with BS4 (known to be heterogeneous) of guinea pigs immunized with antigen $a$, organisms of the BS4R variant appeared to be selected from the challenge population (Parsons et al., 1979). Thus, immunity appeared to have been circumvented by selection of variant gonococci, which emphasizes the difficulty inherent in attempts to immunize against this notoriously variable organism with purified bacterial fractions.

We thank Mrs J. Lichfield and Miss E. Walker for skilled technical assistance. C.W.P. was supported by a Research Fellowship from the Wellcome Trust.

\section{REFERENCES}

Almeida, J. D. \& Waterson, A. P. (1969). The morphology of virus-antibody interaction. $A d$ vances in Virus Research 15, 307-338.

Arko, R. J., Bullard, J. C. \& Duncan, W. P. (1976). Effects of laboratory maintenance on the surface reactive antigens of Neisseria gonorrhoeae. British Journal of Venereal Disease 52, 316-325.

Brinton, C. C., Bryan, J., Dillon, J. A., Guerina, N., Jacobson, L. J., LabiK, A., Lee, S., Levine, A., Lim, S., McMichael, J., Polen, S., Rogers, K., To, A. C. C. \& To, S. C. M. (1978). Uses of pili in gonorrhoea control: role of bacterial pili in disease, purification and properties of gonococcal pili, and progress in the development of a gonococcal pilus vaccine for gonorrhoea. In Immunobiology of Neisseria gonorrhoeae, pp. 155-178. Edited by G. F. Brooks, E. C. Gotschlich, K. K. Holmes, W. D. Sawyer \& F. E. Young. Washington: American Society for Microbiology.

Buchanan, T. M. (1978). Antigen-specific serotyping of Neisseria gonorrhoeae. I. Use of an enzymelinked immunosorbent assay to quantitate pilus antigens on gonococci. Journal of Infectious Diseases 138, 319-325.

Buchanan, T. M., Swanson, J., Holmes, K. K., Kraus, S. J. \& Gotschlich, E. C. (1973). Quantitative determination of antibody to gonococcal pili. Changes in antibody levels with gonococcal infection. Journal of Clinical Investigation 52, 2896-2909.

Buchanan, T. M., Pearce, W. A., Schoolnik, G. K. \& ARko, R. J. (1977). Protection against infection with Neisseria gonorrhoeae by immunization with outer membrane protein complex and purified pili. Journal of Infectious Diseases 136, S132-S137.

JePHCOTt, A. E. \& REYN, A. (1971). Neisseria gonorrhoeae: colony variation I. Acta pathologica et microbiologica scandinavica B79, 609-614.

JephCott, A. E., ReYN, A. \& BirCh-ANDERSEN, A. (1971). Neisseria gonorrhoeae III. Demonstration of presumed appendages to cells from different colony types. Acta pathologica et microbiologica scandinavica B79, 437-439.

Kellogg, D. S., Peacock, W. L., Deacon, W. E., Brown, L. \& Pirkie, C. I. (1963). Neisseria gonorrhoeae I. Virulence genetically linked to clonal variation. Journal of Bacteriology 85, 1274-1279.

LAEMMLI, U. K. (1970). Cleavage of structural proteins during the assembly of the head of bacteriophage T4. Nature, London 227, 680-685.

LAMBden, P. R., Robertson, J. N. \& Watt, P. J. (1980). Biological properties of two distinct pilus types produced by isogenic variants of Neisseria gonorrhoeae P9. Journal of Bacteriology 141, 393-396.

McGee, Z. A., Gross, J., Dourmashkin, R. R. \& TAYLOR-ROBINSON, D. (1976). Nonpilar surface appendages of colony type 1 and colony type 4 gonococci. Infection and Immunity 14, 266-270.

NovotnY, P. \& TuRner, W. H. (1975). Immunological heterogeneity of pili of Neisseria gonorrhoeae. Journal of General Microbiology 89, 87-92.

Novotny, P., Short, J. A. \& Walker, P. D. (1975). An electron microscope study of naturally occurring and cultured cells of Neisseria gonorrhoeae. Journal of Medical Microbiology 8, 413-427.

Parsons, N. J., PenN, C. W., Veale, D. R. \& SMITH, H. (1979). More than one antigen contributes to the immunogenicity of Neisseria gonorrhoeae in the guinea pig chamber model. Journal of General Microbiology 113, 97-104.

Parsons, N. J., PenN, C. W., Veale, D. R. \& SMITH, H. (1980). The complexity of immunogenicity of Neisseria gonorrhoeae in the guinea pig subcutaneous chamber model. Journal of General Microbiology 118, 523-527.

Penn, C. W., Sen, D., Veale, D. R., Parsons, N. J., SMITH, H. \& WITT, K. (1976). Morphological, biological and antigenic properties of Neisseria gonorrhoeae adapted to growth in guinea-pig 
subcutaneous chambers. Journal of General Microbiology 97, 35-43.

Penn, C. W., Veale, D. R. \& Smith, H. (1977). Selection from gonococci grown in vitro of a colony type with some virulence properties of organisms adapted in vivo. Journal of General Microbiology 100, 147-158.

Penn, C. W., Parsons, N. J., Veale, D. R. \& Smith, H. (1978). Correlation with different immunotypes of gonococcal antigens associated with growth in vivo. Journal of General Microbiology 105, 153157.

Penn, C. W., Parsons, N. J., Veale, D. R. \& Smith, $H$. (1979). Antigenic and physical heterogeneity of gonococcal pili. Society for General Microbiology Quarterly 7, 29.

RoBertson, J. N. (1979). Protection by monospecific gonococcal antisera of the chicken embryo challenged with Neisseria gonorrhoeae. Journal of Medical Microbiology 12, 283-289.

Robertson, J. N., VINCENT, P. \& WARD, M. E. (1977). Preparation and properties of gonococcal pili. Journal of General Microbiology 102, 169-178.

SALIT, I. E. \& Gotschlich, E. C. (1978). Gonococcal colour and opacity variants: virulence for chick embryos. Infection and Immunity 22, 359364.
Salit, I. E., Blake, M. \& Gotschlich, E. C. (1980). Intra-strain heterogeneity of gonococcal pili is related to opacity colony variance. Journal of Experimental Medicine 151, 716-725.

Swanson, J., Kraus, S. J. \& Gotschlich, E. C. (1971). Studies on gonococcus infection J. Pili and zones of adhesion: their relation to gonococcal growth patterns. Journal of Experimental Medicine 134, 886-906.

TURNER, W. H. \& NovotNY, P. (1976). The inability of Neisseria gonorrhoeae pili antibodies to confer immunity in subcutaneous guinea pig chambers. Journal of General Microbiology 92, 224-228.

Veale, D. R., Smith, H., Witt, K. A. \& Marshall, R. B. (1975). Differential ability of colonial types of Neisseria gonorrhoeae to produce infection and an inflammatory response in subcutaneous perforated plastic chambers in guinea pigs and rabbits. Journal of Medical Microbiology 8, 325335.

WARD, M. E. \& WATT, P. J. (1971). The preservation of gonococci in liquid nitrogen. Journal of Clinical Pathology 24, 122-123.

Wilson, G. S. \& Miles, A. (1975). In Topley \& Wilson's Principles of Bacteriology, Virology and Immunity, 6th edn, pp. 412-415. London: Edward Arnold. 